\title{
Preface for the Special Issue on Culturally Aware Educational Technologies
}

\author{
Amy Ogan • W. Lewis Johnson
}

Published online: 17 January 2015

(C) International Artificial Intelligence in Education Society 2015

One of the major concerns and hopes for the 21st century has been the education of a global society. The increase in social connectivity through expanding Internet capabilities, the proliferation of computing technologies, and the growing sophistication of educational tools and systems has provided significant promise for achieving this goal. However, technical and conceptual barriers currently stand in the way of realizing this promise. Lingering discrepancies in the utilization of information and communication technology (ICT) around the world impede the worldwide diffusion of advanced educational technologies. Moreover, research in education has shown that teaching methodologies and instructional design often differ from one culture to another, and cannot always be universally applied, as their effects can vary greatly from one context to another. AIED developers therefore must be culturally aware, lest their designs incorporate implicit cultural assumptions that will impede adoption in other cultural contexts. Ideally their designs should be culturally aware as well, so that they are adaptable to a variety of cultural contexts.

Researchers have additionally been interested in cultural modeling and cultural factors for another reason. In a global society, learners' cultural awareness and tolerance of cultural diversity are increasingly important. This presents a new opportunity for learning technologies, which can expose learners in immersive and supportive ways to cultural practices, perspectives, and values that they may not otherwise be able to access. The incorporation of novel technologies such as social media, mobile computing, and online communities can facilitate the sharing of experiences and provide a rich source of cultural diversity for learning interventions embedded in simulations, games, and other rich formats.

However in both of these cases instilling cultural aspects in learning technologies remains a major challenge. Cultural issues are fluid, diverse, and continuously evolve as societies change. They may involve interrelations between such complex factors as

\footnotetext{
A. Ogan

Carnegie Mellon University, 5000 Forbes Avenue, Pittsburgh, PA 15213, USA

e-mail: aeo@andrew.cmu.edu
}

W. L. Johnson $(\bowtie)$

Alelo Inc., 12910 Culver B1., Suite J, Los Angeles, CA 90066, USA

e-mail: ljohnson@alelo.com 
geographic location, religion, class, and even individual personality. In order to address these concerns, we must therefore re-examine the architectures and approaches underlying current learning technologies. The time is ripe to bring together relevant disciplines (e.g., computational modeling, virtual agents, cultural studies, communications, human computer interaction, and learning science) in order to shape the next generation of culture-aware learning technologies. As AIED enters its 25th year it is appropriate that our community tackle these issues.

This special issue on Culturally Aware Educational Technologies provides a view of the state of the art in educational technologies that take into account cultural diversity to meet the needs of a global community of learners. The six papers in this special issue attest to the growing interest in this area. We solicited contributions from researchers and practitioners concerned with issues of representing culture as well as its impact and influence in the domain of AIED and educational technologies. Papers submitted to this special issue situated their work with respect to relevant research in both culture and educational practice, calling on many subfields related to AIED (user modeling and adaptation, knowledge representation, computer-supported collaborative learning, instructional design, serious games, etc.), from the perspective of computer science, psychology, intercultural studies or other related fields.

The first paper (Nye 2014) nicely frames the problem of culture by providing an overview of the challenges and successes of Intelligent Tutoring Systems by and for the developing world. Nye analyzes and describes an issue that is frequently left out of the reporting of studies with such systems: technological barriers that can hinder deployments and may lead to less publishable work in developing nations. Nye notes that technological advances can also accelerate the adoption of new technologies by leapfrogging stages of technological development. We see this as developing countries are embracing mobile technologies instead of desktop computing systems.

The next paper in this issue (Blanchard 2014) is a call for attention to the fact that the vast majority of work in AIED has been done by and for the Western world. In it, Blanchard analyzes ten years of AIED publications with respect to the location of origin of the authors and the student population studied, and discusses the results with a panel of senior AIED researchers who provide commentary on these issues. The main finding of the article could perhaps have been anticipated, given that the development of the field of AIED has been strongly influenced by cognitive psychology, a discipline which has also had this limitation in its body of work. However, there is significant opportunity for this discrepancy to be addressed, as Blanchard (in line with Nye) notes the growing market share in developing countries for educational technologies. The AIED community, whether researchers, reviewers, or the executive committee, should heed the warnings contained in this article, and carefully consider the solutions proposed in order to continue the positive growth of the society.

Taking Blanchard's concerns to heart, the third paper, authored by one of the guest editors of this issue along with an international team (Ogan et al. 2014), is an investigation of the potential for cross-cultural modelling of skills for AIED systems. It compares models of help-seeking skill based on student practice in an intelligent tutoring system in three different contexts: Costa Rica, the Philippines, and a site in the country where the system was originally developed, the United States, and finds that the models are not transferrable across contexts. The authors posit that cultural factors are at least in part responsible for this result, and yet align with Blanchard's warning 
that "Providing assumptions and claims at this stage would thus be likely to end up in the expression of cultural stereotypes, which would be detrimental to this emerging research trend that is obviously in an exploratory phase." Instead, they call for caution in the application of such models to new contexts, and for further research in these areas.

The fourth paper (Mohammed \& Mohan 2014) aims one step beyond modeling, towards adapting the content of an AIED system to be more sensitive to the learner's context. It describes a system that demonstrates an intriguing approach of providing the learners themselves with the ability to adjust the level of contextual specificity, while the system dynamically generates feedback, hints, problem descriptions, and images with grammar and vocabulary that matches that cultural preference. In this way the system gives some control back to the learner, and avoids some of the difficulties that current approaches have in making assumptions about learner background and preferences that may be unwarranted. It models culture as a collection of influences and affiliations to cultural groups, making it possible to model cultural diversity within national learner populations. Deployments of this system additionally provide researchers with the opportunity to experimentally investigate phenomena surrounding the social acceptability of non-dominant language use in education, and its effects on learning.

The fifth paper ( $\mathrm{Si} 2014$ ) addresses the alternate side of cultural awareness, the use of a system to support greater cultural and linguistic awareness for a learner. Specifically, it describes an environment for young children to learn and practice Chinese. This work utilizes a technology that we expect to see more frequently in AIED systems moving forward: a Kinect sensor which allows for a wider variety of input than such systems have typically used, and which may be particularly well suited to domains like culture where both verbal and non-verbal skills (such as proximity or gestures) are of interest for teaching.

The final paper, by an interdisciplinary multinational European team (Hall et al. 2014), is also in the area of cultural instruction. It describes an experiential learning environment in which children learn cultural conflict competence by exploring the interactions between virtual agents representing synthetic cultures. Rather than focusing on novel sensing or input technologies, they instead take a theoretical approach by extending Bennett's Developmental Model of Intercultural Sensitivity (Bennett 1986) into a learning framework for developing intercultural competence. Their results indicate that synthetic cultural environments can promote intercultural competence, when combined with suitable classroom instruction.

The work in this special issue is informed by research in modelling culture and cross-cultural competence, as well as education and computing. This demonstrates an increasing level of maturity and sophistication in this type of work. At the same time this issue illustrates the limitations of our current understanding of culture as it applies to the design of educational technologies. Popular formal models of national cultures, such as the dimensional models of Hofstede et al. (2010) and Hall (1966), might appear to offer promise as a basis for modelling culture in culturally-aware educational technologies. However, the authors of this article did not find them to live up to this potential; as Hall et al. (2014) note, "The Hofstede dimensions are zoomed-out abstractions, derived from aggregated society-wide analysis." It is difficult to take these general cultural characteristics and apply them to specific design problems, such as how to support help-seeking behavior across cultures. On the other hand, richer factorial 
models of culture such as Mohammed and Mohan's offer promise as a way to tailor learning to the cultural background of individual learners. Overall this special issue presents significant steps forward toward the development of a theoretically grounded, model-based approach to educational technologies, that automatically customizes learning experiences to the specific characteristics of learners with diverse cultural backgrounds.

\section{References}

Bennett, M. J. (1986). A developmental approach to training for intercultural sensitivity. International Journal of Intercultural Relations, 10(2), 179-196.

Blanchard, E. (2014). Socio-cultural imbalances in AIED research: investigations, implications, and opportunities. International Journal of Artificial Intelligence in Education. doi:10.1007/s40593-014-0027-7.

Hall, E. T. (1966). The hidden dimension. Garden City: Doubleday.

Hall, L., Tazzyman, S., Hume, C., Endrass, B., Lim, M.-Y., Hofstede, G., Paiva, A., Andre, E., Kappas, A., \& Aylett, R. (2014). Learning to overcome cultural conflict through engaging with intelligent agents in synthetic cultures. International Journal of Artificial Intelligence in Education. doi:10.1007/s40593-014-0031-y.

Hofstede, G., Hofstede, G. J., \& Minkov, M. (2010). Cultures and organizational software of the mind (3rd ed.). New York: McGraw Hill.

Mohammed, P. \& Mohan, P. (2014). Dynamic contextualization of educational context if intelligent learning environments using ICON. International Journal of Artificial Intelligence in Education.

Nye, B. D. (2014). Intelligent tutoring systems by and for the developing world: a review of trends and approaches for educational technology in a global context. International Journal of Artificial Intelligence in Education. doi:10.1007/s40593-014-0028-6.

Ogan, A., Walker, E., Baker, R., Rodrigo, M. T., Soriano, J. C., \& Castro, M. J. (2014). Towards understanding how to assess help-seeking behavior across cultures. International Journal of Artificial Intelligence in Education. doi:10.1007/s40593-014-0034-8.

$\mathrm{Si}, \mathrm{M}$. (2014). A virtual space for children to meet and practice Chinese. International Journal of Artificial Intelligence in Education. 